\title{
An optimal control problem for linear SDE of mean-field type with terminal constraint and partial information
}

Haiyan Zhang ${ }^{1 *}$

"Correspondence:

wszhhaiyan@163.com; wszhhaiyan@sdjtu.edu.cn

'College of Sciences, Shandong

Jiaotong University, Jinan, China

\begin{abstract}
This paper is concerned with an optimal control problem for a linear stochastic differential equation (SDE) of mean-field type, where the drift coefficient of observation equation is linear with respect to the state, the control and their expectations, and the state is subject to a terminal constraint. The control problem cannot be solved by transforming it into a standard optimal control problem for an SDE without mean-field term. By virtue of a backward separation method with a decomposition technique, one optimality condition and one forward-backward filter are derived. Two linear-quadratic (LQ) optimal control problems and one cash management problem with terminal constraint and partial information are studied, and optimal feedback controls are explicitly obtained.
\end{abstract}

Keywords: Feedback control; Filter; LQ optimal control; Necessary condition; SDE of mean-field type

\section{Introduction}

One begins with a complete filtered probability space $\left(\Omega, \mathcal{F},\left(\mathcal{F}_{t}\right)_{0 \leq t \leq T}, \mathbb{P}\right)$, on which are given an $\mathcal{F}_{t}$-adapted standard Brownian motion $\left(\omega_{t}, \tilde{\omega}_{t}\right)$ with value in $\mathbb{R}^{2}$ and a Gaussian random variable $\xi$ with mean $\mu_{0}$ and covariance $\sigma_{0} .(\omega, \tilde{\omega})$ is independent of $\xi$. Let $T>$ 0 be a fixed time horizon, let $\mathbb{R}^{m}$ be the $m$-dimensional Euclidean space, and let $f_{x}$ be the partial derivative of $f$ with respect to $x$. If $x:[0, T] \rightarrow \mathbb{R}$ is uniformly bounded, one writes $x \in L^{\infty}(0, T ; \mathbb{R})$. If $x:[0, T] \rightarrow \mathbb{R}$ is square-integrable, one writes $x \in L^{2}(0, T ; \mathbb{R})$. If $x:[0, T] \times \Omega \rightarrow \mathbb{R}$ is an $\mathcal{F}_{t}$-adapted, square-integrable process, one writes $x \in L_{\mathcal{F}}^{2}(0, T ; \mathbb{R})$. One also adopts similar notations for other filtrations and Euclidean spaces.

Consider the linear SDE

$$
\left\{\begin{array}{l}
d x_{t}^{v}=\left(a_{t} x_{t}^{v}+\bar{a}_{t} \mathbb{E} x_{t}^{v}+b_{t} v_{t}+\bar{b}_{t} \mathbb{E} v_{t}\right) d t+c_{t} d \omega_{t}+\tilde{c}_{t} d \tilde{\omega}_{t} \\
x_{0}^{v}=\xi
\end{array}\right.
$$

where $\mathbb{E}$ is expectation, and $v$ is a control process. Since $\mathbb{E} x_{t}^{v}$ and $\mathbb{E} v_{t}$ appear in the equation, one calls it an SDE of mean-field type. Assume that the solution $x^{v}$ to the equation is

(c) The Author(s) 2019. This article is distributed under the terms of the Creative Commons Attribution 4.0 International License (http://creativecommons.org/licenses/by/4.0/), which permits unrestricted use, distribution, and reproduction in any medium, provided you give appropriate credit to the original author(s) and the source, provide a link to the Creative Commons license, and indicate if changes were made. 
observed through

$$
\left\{\begin{array}{l}
d y_{t}^{v}=\left(f_{t} x_{t}^{v}+\bar{f}_{t} \mathbb{E} x_{t}^{v}+g_{t} v_{t}+\bar{g}_{t} \mathbb{E} v_{t}\right) d t+h_{t} d \tilde{\omega}_{t} \\
y_{0}=0
\end{array}\right.
$$

The cost functional is

$$
\mathcal{J}[v]=\mathbb{E}\left[\int_{0}^{T} l\left(t, x_{t}^{v}, \mathbb{E} x_{t}^{v}, v_{t}, \mathbb{E} v_{t}\right) d t+\phi\left(x_{T}^{v}, \mathbb{E} x_{T}^{v}\right)\right] .
$$

Here $v_{t}$ is required to be $\sigma\left\{y_{s}^{v} ; 0 \leq s \leq t\right\}$-adapted and to satisfy

$$
\mathbb{E} \sup _{0 \leq t \leq T}\left|v_{t}\right|^{2}<+\infty
$$

and

$$
\mathbb{E} \varphi\left(x_{T}^{v}, \mathbb{E} x_{T}^{v}\right)=0
$$

the functions $a, \bar{a}, b, \bar{b}, c, \tilde{c}, f, \bar{f}, g, \bar{g}, h, l, \phi$ and $\varphi$ will be specified in Sect. 2. This is a partially observable optimal control problem with terminal constraint. This problem can reduce to an optimal control problem with certain additional control domain constraint, but it cannot be studied by classical control theory for SDE without mean-field term. From this viewpoint, this problem extends some standard optimal control problems and covers a few financial models.

Classical variation provides an effective tool for studying optimal control problems. However, it is not always valid for partially observed optimal control problems. A main reason is there is a circular dependence between the control $v$ and the observation $y^{v}$. In 2008, Wang and Wu [1] originally proposed a backward separation method. In 2018, Wang coauthored their monograph [2], where the backward separation method was systematically introduced and was regarded as one of most important tools for studying partially observed optimal control problems. Combining the backward separation method with Girsanov's measure transformation, the circular dependence between $v$ and $y^{v}$ was decoupled in Wang et al. [3], and then a necessary condition for optimality was derived. Along this line, Zhang [4], Ma and Liu [5] extended [3] to the case of correlated state and observation noises, and the case of risk-sensitive control, respectively. Buckdahn et al. [6] studied an optimal control problem for SDE of conditional mean-field type. One emphasizes that [3-5] and [6] are based on the assumption that the drift coefficients of observation equations are uniformly bounded with respect to their components, which is restricted in some applications. Using the backward separation method with an approximation technique, Wang et al. [7] generalized [3-6] in the sense that the drift coefficient of observation equation linearly grows with respect to the state, and for any $\ell>0$, the control $v$ satisfies $\mathbb{E} \sup _{0 \leq t \leq T}\left|v_{t}\right|^{\ell}<+\infty$. Note that [7] did not study the case of mean-field and terminal constraint.

Clearly, the control problem in this paper does not satisfy the assumptions above, and then the foregoing techniques are not valid. To overcome the difficulty caused, one will adopt a decomposition technique introduced in Wang et al. [8, 9], where a partially observable forward-backward stochastic control system without mean-field term and/or 
terminal constraint was considered. Combining the decomposition technique with the backward separation method, one solves the control problem. The contributions of this paper are as follows.

- One new necessary condition for optimality is derived. The condition together with forward-backward filter provides an effective method for studying stochastic optimal control with terminal constraint and incomplete information.

- Three LQ examples with terminal constraint and partial information are solved, and optimal feedback controls are obtained by accident.

- An SDE of mean-field type naturally arises from the study of standard LQ optimal control driven by SDE without mean-field term. This interesting contribution can be found in Example 4.2 below.

The control problem is also related to those of Meyer-Brandis et al. [10], Elliott et al. [11], Yong [12], Hafayed and Abbas [13], Ni et al. [14] and Hafayed et al. [15]. Specifically, $[10,15]$, respectively, studied a mean-field type control problem with partial information, where neither noisy observation nor filter is studied. The other work investigated meanfield type controls with complete information.

The rest of this paper is organized as follows. In Sect. 2, one reformulates the control problem and provides preliminary results. Section 3 derives one optimality condition and one forward-backward filtering equation of mean-field type. In Sect. 4, one explicitly solves three LQ optimal control problems with terminal constraint and partial information. Finally, in Sect. 5, one gives some concluding remarks.

\section{Problem formulation and preliminary}

Define $x^{0}$ and $y^{0}$ by two SDEs

$$
\left\{\begin{array}{l}
d x_{t}^{0}=\left(a_{t} x_{t}^{0}+\bar{a}_{t} \mathbb{E} x_{t}^{0}\right) d t+c_{t} d \omega_{t}+\tilde{c}_{t} d \tilde{\omega}_{t}, \\
x_{0}^{0}=\xi
\end{array}\right.
$$

and

$$
\left\{\begin{array}{l}
d y_{t}^{0}=\left(f_{t} x_{t}^{0}+\bar{f}_{t} \mathbb{E} x_{t}^{0}\right) d t+h_{t} d \tilde{\omega}_{t} \\
y_{0}=0
\end{array}\right.
$$

where $a, \bar{a}, c, \tilde{c}, f, \bar{f}, h \in L^{\infty}(0, T ; \mathbb{R})$. Assume that the control $v \in L_{\mathcal{F}}^{2}(0, T ; \mathbb{R})$. Define $x^{v, 1}$ and $y^{v, 1}$ by

$$
\left\{\begin{array}{l}
\dot{x}_{t}^{\nu, 1}=a_{t} x_{t}^{\nu, 1}+\bar{a}_{t} \mathbb{E} x_{t}^{\nu, 1}+b_{t} v_{t}+\bar{b}_{t} \mathbb{E} v_{t}, \\
x_{0}^{v, 1}=0,
\end{array}\right.
$$

and

$$
\left\{\begin{array}{l}
\dot{y}_{t}^{\nu, 1}=f_{t} x_{t}^{\nu, 1}+\bar{f}_{t} \mathbb{E} x_{t}^{\nu, 1}+g_{t} v_{t}+\bar{g}_{t} \mathbb{E} v_{t}, \\
y_{0}^{\nu, 1}=0
\end{array}\right.
$$


where $b, \bar{b}, g, \bar{g} \in L^{\infty}(0, T ; \mathbb{R})$. It is clear that Eqs. (1), (2), (3) and (4) have unique solutions, respectively. Set

$$
x_{t}^{\nu}=x_{t}^{0}+x_{t}^{\nu, 1} \text { and } y_{t}^{v}=y_{t}^{0}+y_{t}^{\nu, 1} .
$$

It follows from Itô's formula that $x^{v}$ and $y^{v}$ satisfy

$$
\left\{\begin{array}{l}
d x_{t}^{v}=\left(a_{t} x_{t}^{v}+\bar{a}_{t} \mathbb{E} x_{t}^{v}+b_{t} v_{t}+\bar{b}_{t} \mathbb{E} v_{t}\right) d t+c_{t} d \omega_{t}+\tilde{c}_{t} d \tilde{\omega}_{t}, \\
x_{0}^{v}=\xi
\end{array}\right.
$$

and

$$
\left\{\begin{array}{l}
d y_{t}^{v}=\left(f_{t} x_{t}^{v}+\bar{f}_{t} \mathbb{E} x_{t}^{v}+g_{t} v_{t}+\bar{g}_{t} \mathbb{E} v_{t}\right) d t+h_{t} d \tilde{\omega}_{t} \\
y_{0}=0
\end{array}\right.
$$

respectively. For any $v \in L_{\mathcal{F}}^{2}(0, T ; \mathbb{R})$, one introduces a constraint condition regarding the terminal state and its distribution

$$
\mathbb{E} \varphi\left(x_{T}^{v}, \mathbb{E} x_{T}^{v}\right)=0
$$

Let

$$
\mathcal{F}_{t}^{y^{0}}=\sigma\left\{y_{s}^{0} ; 0 \leq s \leq t\right\}, \quad \mathcal{F}_{t}^{y^{v}}=\sigma\left\{y_{s}^{v} ; 0 \leq s \leq t\right\},
$$

and let $U$ be a nonempty convex subset of $\mathbb{R}$. Define three admissible control sets

$$
\begin{aligned}
\mathcal{U}_{\mathrm{ad}}^{0}= & \left\{v \mid v_{t} \text { is an } \mathcal{F}_{t}^{y^{0}} \text {-adapted process with value in } U\right. \text { and satisfies } \\
& \left.\mathbb{E} \sup _{0 \leq t \leq T}\left|v_{t}\right|^{2}<+\infty\right\}, \\
\mathcal{U}_{\mathrm{ad}}= & \left\{v \mid v \in \mathcal{U}_{\mathrm{ad}}^{0} \text { is an } \mathcal{F}_{t}^{y^{\nu}} \text {-adapted process }\right\},
\end{aligned}
$$

and

$$
\mathcal{U}_{\mathrm{ad}}^{c}=\left\{v \mid v \in \mathcal{U}_{\mathrm{ad}} \text { satisfies terminal constraint (8) }\right\} .
$$

It is easy to see that the inclusion relationship among them is

$$
\mathcal{U}_{\mathrm{ad}}^{0} \supseteq \mathcal{U}_{\mathrm{ad}} \supseteq \mathcal{U}_{\mathrm{ad}}^{\mathrm{c}}
$$

With (5) and the definition of $\mathcal{U}_{\mathrm{ad}}$, one proves the equality

$$
\mathcal{F}_{t}^{y^{v}}=\mathcal{F}_{t}^{y^{0}}, \quad v \in \mathcal{U}_{\mathrm{ad}}
$$

In fact, since $v_{t}$ is $\mathcal{F}_{t}^{y^{0}}$-adapted, then it follows from (3) and (4) that $y_{t}^{\nu, 1}$ is $\mathcal{F}_{t}^{y^{0}}$-adapted, and thus $y_{t}^{v}$ is also $\mathcal{F}_{t}^{y^{0}}$-adapted by using the second equality in (5). This implies that $\mathcal{F}_{t}^{y^{y}} \subseteq$ $\mathcal{F}_{t}^{y^{0}}, v \in \mathcal{U}_{\text {ad }}$. In the same way, one gets $\mathcal{F}_{t}^{y^{v}} \supseteq \mathcal{F}_{t}^{y^{0}}, v \in \mathcal{U}_{\mathrm{ad}}$. Then one draws the desired conclusion. 
The cost functional is in the form of

$$
\mathcal{J}[v]=\mathbb{E}\left[\int_{0}^{T} l\left(t, x_{t}^{v}, \mathbb{E} x_{t}^{v}, v_{t}, \mathbb{E} v_{t}\right) d t+\phi\left(x_{T}^{v}, \mathbb{E} x_{T}^{v}\right)\right]
$$

where $l:[0, T] \times \mathbb{R}^{2} \times U \times \mathbb{R} \rightarrow \mathbb{R}$ and $\phi, \varphi: \mathbb{R}^{2} \rightarrow \mathbb{R}$ are continuously differentiable with respect to $(x, \bar{x}, v, \bar{v})$ and $(x, \bar{x})$, respectively, and there is a constant $C>0$ such that

$$
\begin{aligned}
& |\psi(x, \bar{x})| \leq C\left(1+|x|^{2}+|\bar{x}|^{2}\right), \\
& \left|\psi_{\chi}(x, \bar{x})\right| \leq C(1+|x|+|\bar{x}|), \\
& |l(t, x, \bar{x}, v, \bar{v})| \leq C\left(1+|x|^{2}+|\bar{x}|^{2}+|v|^{2}+|\bar{v}|^{2}\right), \\
& \left|l_{\chi}(t, x, \bar{x}, v, \bar{v})\right| \leq C(1+|x|+|\bar{x}|+|v|+|\bar{v}|),
\end{aligned}
$$

with $\psi=\phi, \varphi$ and $\chi=x, \bar{x}, v, \bar{v}$.

Then the optimal control problem with terminal constraint is restated as follows.

Problem (TC) Find a $u \in \mathcal{U}_{\text {ad }}^{c}$ such that

$$
\mathcal{J}[u]=\inf _{v \in \mathcal{U}_{\mathrm{ad}}^{c}} \mathcal{J}[v]
$$

subject to (6), (7), (8) and (9). Any $u$ satisfying the equality is called an optimal control of Problem (TC), and $x^{u}$ is called the optimal state corresponding to $u$.

One also introduces an auxiliary problem without terminal constraint.

Problem (A) Find a $u \in \mathcal{U}_{\text {ad }}$ such that

$$
J_{\kappa}[u]=\inf _{v \in \mathcal{U}_{\mathrm{ad}}} J_{\kappa}[v]
$$

subject to (6), (7) and

$$
J_{\kappa}[v]=\mathbb{E}\left[\int_{0}^{T} l\left(t, x_{t}^{v}, \mathbb{E} x_{t}^{v}, v_{t}, \mathbb{E} v_{t}\right) d t+\phi\left(x_{T}^{v}, \mathbb{E} x_{T}^{v}\right)+\kappa \varphi\left(x_{T}^{v}, \mathbb{E} x_{T}^{v}\right)\right], \quad \kappa \in \Delta \subseteq \mathbb{R} .
$$

In what follows, one provides two preliminary results, whose proofs can be found in the Appendix.

Proposition 2.1 For any $\kappa \in \Delta$, one has

$$
\inf _{v^{\prime} \in \mathcal{U}_{\mathrm{ad}}} J_{\kappa}\left[v^{\prime}\right]=\inf _{v \in \mathcal{U}_{\mathrm{ad}}^{0}} J_{\kappa}[v]
$$

Proposition 2.2 Suppose that, for any $\kappa \in \Delta, u_{\kappa}$ is an optimal control of Problem (A). Moreover, suppose that there exists $\kappa_{0} \in \Delta$ such that

$$
\varphi\left(x_{T}^{u_{\kappa_{0}}}, \mathbb{E} x_{T}^{u_{\kappa_{0}}}\right)=0
$$

Then $u=u_{\kappa_{0}}$ is an optimal control of Problem (TC). 
Proposition 2.1 reveals the equivalence between Problem (A) and the problem of minimizing $J_{\kappa}[v]$ over $\mathcal{U}_{\text {ad }}^{0}$. Proposition 2.2 together with Proposition 2.1 shows that one can obtain an optimal control of Problem (TC) by the following procedures: (1) to derive all optimal controls $u_{\kappa}$ of Problem (A); (2) to find $u_{\kappa_{0}}$ satisfying $\mathbb{E} \varphi\left(x_{T}^{u_{\kappa_{0}}}, \mathbb{E} x_{T}^{u_{\kappa_{0}}}\right)=0$. Then such $u_{\kappa_{0}}$ is exactly an optimal control of Problem (TC). Clearly, it is a more convenient approach in at least some detailed cases. See, e.g., Sect. 4 for more details.

This remark shows that the second procedure above can easily be finished in general, and thus it is enough to study Problem (A).

\section{Optimality condition of Problem (A)}

For any $v, v_{j} \in \mathcal{U}_{\mathrm{ad}}$, let $x^{v}$ and $x^{v_{j}}$ be the solutions to (6) corresponding to $v$ and $v_{j}, j=1,2, \ldots$ For simplicity, we set

$$
\begin{aligned}
& \left(\Upsilon_{t}^{\theta}\right)=\left(t, x_{t}^{\nu}+\theta\left(x_{t}^{v_{j}}-x_{t}^{\nu}\right), \mathbb{E}\left(x_{t}^{\nu}+\theta\left(x_{t}^{v_{j}}-x_{t}^{\nu}\right)\right), v_{t}+\theta\left(v_{j, t}-v_{t}\right), \mathbb{E}\left(v_{t}+\theta\left(v_{j, t}-v_{t}\right)\right)\right), \\
& \left(\Theta_{t}^{\lambda}\right)=\left(t, x_{t}^{\lambda}, \mathbb{E} x_{t}^{\lambda}, \lambda_{t}, \mathbb{E} \lambda_{t}\right), \quad\left(\Xi_{t}^{\lambda}\right)=\left(x_{t}^{\lambda}, \mathbb{E} x_{t}^{\lambda}\right),
\end{aligned}
$$

where $\lambda=v, u_{\kappa}, v_{j}, j=1,2, \ldots$

Theorem 3.1 If $u_{\kappa}$ is an optimal control of Problem (A), then the backward stochastic differential equation of mean-field type

$$
\left\{\begin{array}{l}
-d p_{\kappa, t}=\left[a_{t} p_{\kappa, t}+l_{x}\left(\Theta_{t}^{u_{\kappa}}\right)+\mathbb{E}\left(\bar{a}_{t} p_{\kappa, t}+l_{\bar{x}}\left(\Theta_{t}^{u_{\kappa}}\right)\right)\right] d t-q_{\kappa, t} d \omega_{t}-\tilde{q}_{\kappa, t} d \tilde{\omega}_{t}, \\
p_{\kappa, T}=\phi_{x}\left(\Xi_{T}^{u_{\kappa}}\right)+\kappa \varphi_{x}\left(\Xi_{T}^{u_{\kappa}}\right)+\mathbb{E}\left(\phi_{\bar{x}}\left(\Xi_{T}^{u_{\kappa}}\right)+\kappa \varphi_{\bar{x}}\left(\Xi_{T}^{u_{\kappa}}\right)\right),
\end{array}\right.
$$

has a unique solution $\left(p_{\kappa}, q_{\kappa}, \tilde{q}_{\kappa}\right) \in L_{\mathcal{F}}^{2}\left(0, T ; \mathbb{R}^{3}\right)$ such that for any $v \in U$

$$
\begin{aligned}
\mathbb{E} & {\left[\left(H_{v}\left(\Theta_{t}^{u_{\kappa}} ; p_{\kappa, t}, q_{\kappa, t}, \tilde{q}_{\kappa, t}\right)\right.\right.} \\
& \left.\left.+\mathbb{E} H_{\bar{v}}\left(\Theta_{t}^{u_{\kappa}} ; p_{\kappa, t}, q_{\kappa, t}, \tilde{q}_{\kappa, t}\right)\right)\left(v-u_{\kappa, t}\right) \mid \mathcal{F}_{t}^{y^{u_{\kappa}}}\right] \geq 0, \quad \kappa \in \Delta,
\end{aligned}
$$

where the Hamiltonian function $H$ is defined by

$$
H(t, x, \bar{x}, v, \bar{v} ; p, q, \tilde{q})=\left(a_{t} x+\bar{a}_{t} \bar{x}+b_{t} v+\bar{b}_{t} \bar{v}\right) p+c_{t} q+\tilde{c}_{t} \tilde{q}+l(t, x, \bar{x}, v, \bar{v})
$$

Proof If $u_{\kappa}$ is an optimal control of Problem (A), Proposition 2.1 implies that

$$
J_{\kappa}\left[u_{\kappa}\right]=\inf _{v \in \mathcal{U}_{\mathrm{ad}}^{0}} J_{\kappa}[v]
$$

For any $v \in \mathcal{U}_{\text {ad }}^{0}$, let $x^{u_{\kappa}+\varepsilon v}$ be the solution to (6) corresponding to $u_{\kappa}+\varepsilon v$, where $0 \leq \varepsilon \leq 1$. Introduce the variational equation

$$
\left\{\begin{array}{l}
\dot{x}_{1, t}=a_{t} x_{1, t}+\bar{a}_{t} \mathbb{E} x_{1, t}+b_{t} v_{t}+\bar{b}_{t} \mathbb{E} v_{t} \\
x_{1,0}=0
\end{array}\right.
$$


which admits a unique solution $x_{1} \in L_{\mathcal{F}}^{2}(0, T ; \mathbb{R})$. It follows from Hölder's inequality that

$$
\lim _{\varepsilon \rightarrow 0} \mathbb{E} \sup _{0 \leq t \leq T}\left|\frac{1}{\varepsilon}\left(x_{t}^{u_{\kappa}+\varepsilon v}-x_{t}^{u_{\kappa}}\right)-x_{1, t}\right|^{2}=0 .
$$

Combining the limit with the optimality of $u$, one derives the first-order variational inequality

$$
\begin{aligned}
0 \leq & \lim _{\varepsilon \rightarrow 0} \frac{J\left[u_{\kappa}+\varepsilon v\right]-J\left[u_{\kappa}\right]}{\varepsilon} \\
= & \mathbb{E} \int_{0}^{T}\left(l_{x}\left(\Theta_{t}^{u_{\kappa}}\right) x_{1, t}+l_{\bar{x}}\left(\Theta_{t}^{u_{\kappa}}\right) \mathbb{E} x_{1, t}+l_{\nu}\left(\Theta_{t}^{u_{\kappa}}\right) v_{t}+l_{\bar{v}}\left(\Theta_{t}^{u_{\kappa}}\right) \mathbb{E} v_{t}\right) d t \\
& +\mathbb{E}\left[\left(\phi_{x}\left(\Xi_{T}^{u_{\kappa}}\right)+\kappa \varphi_{x}\left(\Xi_{T}^{u_{\kappa}}\right)\right) x_{1, T}+\left(\phi_{\bar{x}}\left(\Xi_{T}^{u_{\kappa}}\right)+\kappa \varphi_{\bar{x}}\left(\Xi_{T}^{u_{\kappa}}\right)\right) \mathbb{E} x_{1, T}\right] .
\end{aligned}
$$

On the other hand, once $x^{u_{\kappa}}$ is determined by (6), (11) admits a unique solution $\left(p_{\kappa}, q_{\kappa}, q_{\kappa}\right)$ in $L_{\mathcal{F}}^{2}\left(0, T ; \mathbb{R}^{3}\right)$. Using Itô's formula to $x_{1} p_{\kappa}$ and inserting it into the variational inequality, one gets

$$
\mathbb{E} \int_{0}^{T}\left[b_{t} p_{\kappa, t}+l_{v}\left(\Theta_{t}^{u_{\kappa}}\right)+\mathbb{E}\left(\bar{b}_{t} p_{\kappa, t}+l_{\bar{v}}\left(\Theta_{t}^{u_{\kappa}}\right)\right)\right] v_{t} d t \geq 0 .
$$

Due to $u_{\kappa} \in \mathcal{U}_{\mathrm{ad}}^{0}$ and the arbitrariness of $v_{t}$, one deduces

$$
\mathbb{E}\left\{\left[b_{t} p_{\kappa, t}+l_{v}\left(\Theta_{t}^{u_{\kappa}}\right)+\mathbb{E}\left(\bar{b}_{t} p_{\kappa, t}+l_{\bar{v}}\left(\Theta_{t}^{u_{\kappa}}\right)\right)\right]\left(v-u_{\kappa, t}\right) \mid \mathcal{F}_{t}^{y^{0}}\right\} \geq 0, \quad \text { for any } v \in U \text {. }
$$

Recalling for any $u_{\kappa} \in \mathcal{U}_{\mathrm{ad}}, \mathcal{F}_{t}^{y^{u_{\kappa}}}=\mathcal{F}_{t}^{y^{0}}$, then one draws the desired conclusion.

According to (12), one needs to compute the optimal filters of (11) and (6) depending on $\mathcal{F}_{t}^{y^{\nu}}$ in order to compute $u_{\kappa}$. For this purpose, one denotes by

$$
\hat{\Phi}_{t}=\mathbb{E}\left[\Phi_{t} \mid \mathcal{F}_{t}^{y^{\nu}}\right] \quad \text { with } \Phi_{t}=x_{t}^{0}, x_{t}^{v}, v \in \mathcal{U}_{\mathrm{ad}}
$$

and

$$
\hat{\Psi}_{t}=\mathbb{E}\left[\Psi_{t} \mid \mathcal{F}_{t}^{y^{u_{\kappa}}}\right] \quad \text { with } \Psi_{t}=p_{\kappa, t}, x_{t}^{u_{\kappa}} p_{\kappa, t}, \tilde{z}_{t}^{u_{\kappa}}
$$

the filters of $\Phi_{t}$ and $\Psi_{t}$, respectively. Moreover, one denotes by

$$
\Sigma_{t}=\mathbb{E}\left(x_{t}^{v}-\hat{x}_{t}^{v}\right)^{2}
$$

the mean square error of $\hat{x}_{t}^{v}, v \in \mathcal{U}_{\mathrm{ad}}$. Using Theorems 2.1 and 2.2 in [2] to (6), (7) and (11), one derives the filters $\hat{x}_{t}^{v}$ and $\hat{p}_{\kappa, t}$ of $x_{t}^{v}$ and $p_{\kappa, t}$ with respect to $\mathcal{F}_{t}^{y^{v}}$.

Theorem 3.2 For any $v \in \mathcal{U}_{\mathrm{ad}}$, the filters $\hat{x}_{t}^{v}$ and $\hat{p}_{\kappa, t}$ satisfy

$$
\left\{\begin{array}{l}
d \hat{x}_{t}^{v}=\left(a_{t} \hat{x}_{t}^{v}+\bar{a}_{t} \mathbb{E} x_{t}^{v}+b_{t} v_{t}+\bar{b}_{t} \mathbb{E} v_{t}\right) d t+\left(\tilde{c}_{t}+\Sigma_{t} f_{t} h_{t}^{-1}\right) d \hat{\omega}_{t}, \\
\hat{x}_{0}^{v}=\mu_{0},
\end{array}\right.
$$


and

$$
\left\{\begin{array}{l}
-d \hat{p}_{\kappa, t}=\left\{a_{t} \hat{p}_{\kappa, t}+\mathbb{E}\left[l_{x}\left(\Theta_{t}^{u_{\kappa}}\right) \mid \mathcal{F}_{t}^{y^{u_{\kappa}}}\right]+\mathbb{E}\left(\bar{a}_{t} p_{\kappa, t}+l_{\bar{x}}\left(\Theta_{t}^{u_{\kappa}}\right)\right)\right\} d t-Q_{t} d \hat{\omega}_{t}, \\
\hat{p}_{\kappa, T}=\mathbb{E}\left[\phi_{x}\left(\Xi_{T}^{u_{\kappa}}\right)+\kappa \varphi_{x}\left(\Xi_{T}^{u_{\kappa}}\right) \mid \mathcal{F}_{T}^{y^{u}}\right]+\mathbb{E}\left(\phi_{\bar{x}}\left(\Xi_{T}^{u_{\kappa}}\right)+\kappa \varphi_{\bar{x}}\left(\Xi_{T}^{u_{\kappa}}\right)\right),
\end{array}\right.
$$

respectively, where $\Sigma$ is the unique solution to

$$
\begin{aligned}
& \left\{\begin{array}{l}
\dot{\Sigma}_{t}-2 a_{t} \Sigma_{t}+\left(\tilde{c}_{t}+\Sigma_{t} f_{t} h_{t}^{-1}\right)^{2}-\left(c_{t}+\tilde{c}_{t}\right)^{2}=0, \\
\Sigma_{0}=\sigma_{0},
\end{array}\right. \\
& \hat{\omega}_{t}=\int_{0}^{t} h_{s}^{-1}\left[d y_{s}^{0}-\left(f_{s} \hat{x}_{s}^{0}+\bar{f}_{s} \mathbb{E} x_{s}^{0}\right) d s\right],
\end{aligned}
$$

is a standard Brownian motion with value in $\mathbb{R}$, and

$$
\left.Q_{t}=\hat{\tilde{z}}_{t}^{u_{\kappa}}+\widehat{\left(\widehat{x_{t}^{u_{\kappa}} p_{\kappa, t}}\right.}-\hat{x}_{t}^{u_{\kappa}} \hat{p}_{\kappa, t}\right) f_{t} h_{t}^{-1}
$$

One emphasizes that (13) with (14) is a forward-backward stochastic differential filtering equation of mean-field type, which has a unique solution $\left(\hat{x}^{u_{\kappa}}, \hat{p}_{\kappa}, Q\right) \in L_{\mathcal{F}^{y^{u_{\kappa}}}}^{2}\left(0, T ; \mathbb{R}^{3}\right)$ for given $u_{\kappa}$. It shows that Theorem 3.2 is different from the usual filtering theories. See, e.g., Xiong [16].

\section{Three LQ cases of Problem (TC)}

In this section, one aims at illustrating Theorems 3.1 and 3.2 by three examples. For convenience, one still adopts the state equation, the observation equation, and the corresponding assumptions introduced in Sects. 2 and 3 unless noted otherwise.

Example 4.1 Find an admissible control to minimize

$$
\begin{aligned}
\mathcal{J}[v]= & \frac{1}{2} \mathbb{E}\left\{\int_{0}^{T}\left[A_{t}\left(x_{t}^{v}\right)^{2}+\bar{A}_{t}\left(\mathbb{E} x_{t}^{\nu}\right)^{2}+B_{t} v_{t}^{2}+\bar{B}_{t}\left(\mathbb{E} v_{t}\right)^{2}\right] d t\right. \\
& \left.+D\left(x_{T}^{v}\right)^{2}+\bar{D}\left(\mathbb{E} x_{T}^{v}\right)^{2}\right\}
\end{aligned}
$$

over $\mathcal{U}_{\text {ad }}^{c}$ with $U=\mathbb{R}$ and the terminal constraint

$$
\mathbb{E} x_{T}^{v}=\gamma, \quad \gamma \in \mathbb{R}
$$

subject to

$$
\left\{\begin{array}{l}
d x_{t}^{v}=\left(a_{t} x_{t}^{v}+\bar{a}_{t} \mathbb{E} x_{t}^{v}+b_{t} v_{t}+\bar{b}_{t} \mathbb{E} v_{t}\right) d t+c_{t} d \omega_{t}+\tilde{c}_{t} d \tilde{\omega}_{t}, \\
x_{0}^{v}=\xi
\end{array}\right.
$$

and

$$
\left\{\begin{array}{l}
d y_{t}^{v}=\left(f_{t} x_{t}^{v}+\bar{f}_{t} \mathbb{E} x_{t}^{v}+g_{t} v_{t}+\bar{g}_{t} \mathbb{E} v_{t}\right) d t+h_{t} d \tilde{\omega}_{t} \\
y_{0}=0
\end{array}\right.
$$


where $A, \bar{A}, B, \bar{B} \in L^{\infty}(0, T ; \mathbb{R}), A_{t}>0, A_{t}+\bar{A}_{t} \geq 0, B_{t}>0, B_{t}+\bar{B}_{t}>0, D \geq 0, D+\bar{D} \geq 0$, $b \neq 0$ and $b+\bar{b} \neq 0$.

Define an auxiliary cost functional without terminal constraint

$$
\mathcal{J}_{\kappa}[v]=J_{\kappa}[v]-\kappa \gamma
$$

with

$$
\begin{aligned}
J_{\kappa}[v]= & \frac{1}{2} \mathbb{E}\left\{\int_{0}^{T}\left[A_{t}\left(x_{t}^{v}\right)^{2}+\bar{A}_{t}\left(\mathbb{E} x_{t}^{v}\right)^{2}+B_{t} v_{t}^{2}+\bar{B}_{t}\left(\mathbb{E} v_{t}\right)^{2}\right] d t\right. \\
& \left.+D\left(x_{T}^{v}\right)^{2}+\bar{D}\left(\mathbb{E} x_{T}^{v}\right)^{2}+2 \kappa x_{T}^{v}\right\}, \quad \kappa \in \Delta .
\end{aligned}
$$

Since both $\kappa$ and $\gamma$ are constants, it is enough to minimize (21) over $\mathcal{U}_{\text {ad }}$ subject to (19) and (20). One will use three steps to explicitly solve the example.

Step 1 Candidate optimal control of the auxiliary problem without terminal constraint. With the data, the Hamiltonian function is

$$
\begin{aligned}
H\left(t, x, \bar{x}, v ; p_{\kappa}, q_{\kappa}, \tilde{q}_{\kappa}\right)= & \left(a_{t} x+\bar{a}_{t} \bar{x}+b_{t} v+\bar{b}_{t} \bar{v}\right) p_{\kappa}+c_{t} q_{\kappa}+\tilde{c}_{t} \tilde{q}_{\kappa} \\
& +\frac{1}{2}\left[A_{t} x^{2}+\bar{A}_{t}(\bar{x})^{2}+B_{t} v^{2}+\bar{B}_{t}(\bar{v})^{2}\right]
\end{aligned}
$$

where $\left(p_{\kappa}, q_{\kappa}, \tilde{q}_{\kappa}\right)$ is determined by the Hamiltonian system

$$
\left\{\begin{array}{l}
d x_{t}^{u_{\kappa}}=\left(a_{t} x_{t}^{u_{\kappa}}+\bar{a}_{t} \mathbb{E} x_{t}^{u_{\kappa}}+b_{t} u_{\kappa, t}+\bar{b}_{t} \mathbb{E} u_{\kappa, t}\right) d t+c_{t} d \omega_{t}+\tilde{c}_{t} d \tilde{\omega}_{t}, \\
-d p_{\kappa, t}=\left[a_{t} p_{\kappa, t}+A_{t} x_{t}^{u}+\mathbb{E}\left(\bar{a}_{t} p_{\kappa, t}+\bar{A}_{t} x_{t}^{u}\right)\right]-q_{\kappa, t} d \omega_{t}-\tilde{q}_{\kappa, t} d \tilde{\omega}_{t} \\
x_{0}^{u_{\kappa}}=\xi, \quad p_{\kappa, T}=D x_{T}^{u_{\kappa}}+\bar{D} \mathbb{E} x_{T}^{u_{\kappa}}+\kappa .
\end{array}\right.
$$

If $u_{\kappa}$ is an optimal control of the auxiliary problem, then it follows from Theorem 3.1 that

$$
B_{t} u_{\kappa, t}+b_{t} \hat{p}_{\kappa, t}+\bar{B}_{t} \mathbb{E} u_{\kappa, t}+\bar{b}_{t} \mathbb{E} p_{\kappa, t}=0
$$

Solving it, we get

$$
u_{\kappa, t}=-B_{t}^{-1}\left\{b_{t} \hat{p}_{\kappa, t}+\left[\bar{b}_{t}-\bar{B}_{t}\left(B_{t}+\bar{B}_{t}\right)^{-1}\left(b_{t}+\bar{b}_{t}\right)\right] \mathbb{E} p_{\kappa, t}\right\} .
$$

Step 2 Feedback form of (23).

Inserting (23) into (22) and taking expectations, one gets an ordinary differential equation

$$
\left\{\begin{array}{l}
\frac{d}{d t} \mathbb{E} x_{t}^{u_{\kappa}}=\left(a_{t}+\bar{a}_{t}\right) \mathbb{E} x_{t}^{u_{\kappa}}-\left(B_{t}+\bar{B}_{t}\right)^{-1}\left(b_{t}+\bar{b}_{t}\right)^{2} \mathbb{E} p_{\kappa, t}, \\
\frac{d}{d t} \mathbb{E} p_{\kappa, t}=-\left(A_{t}+\bar{A}_{t}\right) \mathbb{E} x_{t}^{u_{\kappa}}-\left(a_{t}+\bar{a}_{t}\right) \mathbb{E} p_{\kappa, t}, \\
\mathbb{E} x_{0}^{u_{\kappa}}=\mu_{0}, \quad \mathbb{E} p_{\kappa, T}=(D+\bar{D}) \mathbb{E} x_{T}^{u_{\kappa}}+\kappa
\end{array}\right.
$$

Note that the first equation and the second equation in (24) are coupled. Since

$$
-\left(B_{t}+\bar{B}_{t}\right)^{-1}\left(b_{t}+\bar{b}_{t}\right)^{2}<0
$$


the assumption condition of Theorem 2.6 in Peng and Wu [17] is satisfied, and hence (24) has a unique solution $\left(\mathbb{E} x^{u_{\kappa}}, \mathbb{E} p_{\kappa}\right)$. Noticing the terminal condition of (24), one sets

$$
\mathbb{E} p_{\kappa, t}=\alpha_{t} \mathbb{E} x_{t}^{u_{\kappa}}+\beta_{\kappa, t},
$$

where $\alpha$ and $\beta_{\kappa}$ are deterministic and differential functions such that $\alpha_{T}=D+\bar{D}$ and $\beta_{\kappa, T}=\kappa$. Using the chain rule for computing the derivative of (25), one has

$$
\begin{aligned}
\frac{d}{d t} \mathbb{E} p_{\kappa, t}= & \dot{\alpha}_{t} \mathbb{E} x_{t}^{u_{\kappa}}+\alpha_{t} \frac{d}{d t} \mathbb{E} x_{t}^{u_{\kappa}}+\dot{\beta}_{\kappa, t} \\
= & {\left[\dot{\alpha}_{t}+\left(a_{t}+\bar{a}_{t}\right) \alpha_{t}-\left(B_{t}+\bar{B}_{t}\right)^{-1}\left(b_{t}+\bar{b}_{t}\right)^{2} \alpha_{t}^{2}\right] \mathbb{E} x_{t}^{u_{\kappa}} } \\
& +\dot{\beta}_{\kappa, t}-\alpha_{t}\left(B_{t}+\bar{B}_{t}\right)^{-1}\left(b_{t}+\bar{b}_{t}\right)^{2} \beta_{\kappa, t} .
\end{aligned}
$$

Comparing the equality with the second equation in (24), one deduces

$$
\left\{\begin{array}{l}
\dot{\alpha}_{t}+2\left(a_{t}+\bar{a}_{t}\right) \alpha_{t}-\left(B_{t}+\bar{B}_{t}\right)^{-1}\left(b_{t}+\bar{b}_{t}\right)^{2} \alpha_{t}^{2}+A_{t}+\bar{A}_{t}=0 \\
\alpha_{T}=D+\bar{D}
\end{array}\right.
$$

and

$$
\left\{\begin{array}{l}
\dot{\beta}_{\kappa, t}+\left[a_{t}+\bar{a}_{t}-\left(B_{t}+\bar{B}_{t}\right)^{-1}\left(b_{t}+\bar{b}_{t}\right)^{2} \alpha_{t}\right] \beta_{\kappa, t}=0, \\
\beta_{\kappa, T}=\kappa
\end{array}\right.
$$

It is easy to see (26) and (27) admit unique solutions, respectively. Inserting (25) into the first equation of (24), one derives

$$
\left\{\begin{aligned}
\frac{d}{d t} \mathbb{E} x_{t}^{u_{\kappa}}= & {\left[a_{t}+\bar{a}_{t}-\left(B_{t}+\bar{B}_{t}\right)^{-1}\left(b_{t}+\bar{b}_{t}\right)^{2} \alpha_{t}\right] \mathbb{E} x_{t}^{u_{\kappa}} } \\
& -\left(B_{t}+\bar{B}_{t}\right)^{-1}\left(b_{t}+\bar{b}_{t}\right)^{2} \beta_{\kappa, t}, \\
\mathbb{E} x_{0}^{u}=\mu_{0} . &
\end{aligned}\right.
$$

Using Theorem 3.2 to (22) with (23), one gets a forward-backward stochastic differential filtering equation of mean-field type,

$$
\left\{\begin{aligned}
& d \hat{x}_{t}^{u_{\kappa}}= {\left[a_{t} \hat{x}_{t}^{u_{\kappa}}-b_{t}^{2} B_{t}^{-1} \hat{p}_{\kappa, t}+\bar{a}_{t} \mathbb{E} x_{t}^{u_{\kappa}}-\left(B_{t}+\bar{B}_{t}\right)^{-1}\left(\bar{b}_{t}^{2}+2 b_{t} \bar{b}_{t}-B_{t}^{-1} \bar{B}_{t} b_{t}^{2}\right) \mathbb{E} p_{\kappa, t}\right] d t } \\
&+\left(\tilde{c}_{t}+\Sigma_{t} f_{t} h_{t}^{-1}\right) d \hat{\omega}_{t}, \\
&-d \hat{p}_{\kappa, t}= {\left[A_{t} \hat{x}_{t}^{u_{\kappa}}+a_{t} \hat{p}_{\kappa, t}+\mathbb{E}\left(\bar{a}_{t} p_{\kappa, t}+\bar{A}_{t} x_{t}^{u_{\kappa}}\right)\right] d t-Q_{t} d \hat{\omega}_{t}, } \\
& \hat{x}_{0}^{u_{\kappa}}=\mu_{0}, \quad \hat{p}_{\kappa, T}=D \hat{x}_{T}^{u_{\kappa}}+\bar{D} \mathbb{E} x_{T}^{u_{\kappa}}+\kappa,
\end{aligned}\right.
$$

where $\Sigma$ and $\hat{\omega}$ satisfy (15) with (16), and $\mathbb{E} x^{u_{\kappa}}$ and $\mathbb{E} p_{\kappa}$ solve (28) and (25), respectively. Since $b \neq 0,(29)$ admits a unique solution $\left(\hat{x}^{u_{\kappa}}, \hat{p}_{\kappa}, Q\right) \in L_{\mathcal{F}^{u_{\kappa}}}^{2}\left(0, T ; \mathbb{R}^{3}\right)$ by Theorem 2.6 in [17] again. Similarly, let

$$
\hat{p}_{\kappa, t}=\Gamma_{t} \hat{x}_{t}^{u_{\kappa}}+\bar{\Gamma}_{t} \mathbb{E} \hat{x}_{t}^{u_{\kappa}}+\Lambda_{\kappa, t},
$$


where $\Gamma, \bar{\Gamma}$ and $\Lambda_{\kappa}$ are three deterministic and differential functions satisfying $\Gamma_{T}=D$, $\bar{\Gamma}_{T}=\bar{D}$ and $\Lambda_{\kappa, T}=\kappa$. It follows from Itô's formula that

$$
\begin{aligned}
d \hat{p}_{\kappa, t}= & \dot{\Gamma}_{t} \hat{x}_{t}^{u_{\kappa}} d t+\Gamma_{t} d \hat{x}_{t}^{u_{\kappa}}+\dot{\bar{\Gamma}}_{t} \mathbb{E} \hat{x}_{t}^{u_{\kappa}} d t+\bar{\Gamma}_{t} d \mathbb{E} \hat{x}_{t}^{u_{\kappa}}+\dot{\Lambda}_{\kappa, t} d t \\
= & \left(\dot{\Gamma}_{t}+a_{t} \Gamma_{t}-B_{t}^{-1} b_{t}^{2} \Gamma_{t}^{2}\right) \hat{x}_{t}^{u_{\kappa}} d t \\
& +\left\{\dot{\bar{\Gamma}}_{t}+\left[a_{t}+\bar{a}_{t}-2\left(B_{t}+\bar{B}_{t}\right)^{-1}\left(b_{t}+\bar{b}_{t}\right)^{2} \Gamma_{t}\right] \bar{\Gamma}_{t}-\left(B_{t}+\bar{B}_{t}\right)^{-1}\left(b_{t}+\bar{b}_{t}\right)^{2} \bar{\Gamma}_{t}^{2}\right. \\
& \left.-\left(B_{t}+\bar{B}_{t}\right)^{-1}\left(\bar{b}_{t}^{2}+2 b_{t} \bar{b}_{t}-B_{t}^{-1} \bar{B}_{t} b_{t}^{2}\right) \Gamma_{t}^{2}+\bar{a}_{t} \Gamma_{t}\right\} \mathbb{E} \hat{x}_{t}^{u_{\kappa}} d t \\
& +\left\{\dot{\Lambda}_{\kappa, t}-\left(\Gamma_{t}+\bar{\Gamma}_{t}\right)\left(B_{t}+\bar{B}_{t}\right)^{-1}\left(b_{t}+\bar{b}_{t}\right)^{2} \Lambda_{\kappa, t}\right\} d t \\
& +\Gamma_{t}\left(\tilde{c}_{t}+\Sigma_{t} f_{t} h_{t}^{-1}\right) d \hat{\omega}_{t} .
\end{aligned}
$$

Comparing it with the second equation in (29), one deduces

$$
\begin{aligned}
& \left\{\begin{array}{l}
\dot{\Gamma}_{t}+2 a_{t} \Gamma_{t}-B_{t}^{-1} b_{t}^{2} \Gamma_{t}^{2}+A_{t}=0 \\
\Gamma_{T}=D
\end{array}\right. \\
& \left\{\begin{array}{c}
\dot{\bar{\Gamma}}_{t}+2\left[a_{t}+\bar{a}_{t}-\left(B_{t}+\bar{B}_{t}\right)^{-1}\left(b_{t}+\bar{b}_{t}\right)^{2} \Gamma_{t}\right] \bar{\Gamma}_{t}-\left(B_{t}+\bar{B}_{t}\right)^{-1}\left(b_{t}+\bar{b}_{t}\right)^{2} \bar{\Gamma}_{t}^{2} \\
\quad-\left(B_{t}+\bar{B}_{t}\right)^{-1}\left(\bar{b}_{t}^{2}+2 b_{t} \bar{b}_{t}-B_{t}^{-1} \bar{B}_{t} b_{t}^{2}\right) \Gamma_{t}^{2}+2 \bar{a}_{t} \Gamma_{t}+\bar{A}_{t}=0 \\
\bar{\Gamma}_{T}=\bar{D}
\end{array}\right.
\end{aligned}
$$

and

$$
\left\{\begin{array}{l}
\dot{\Lambda}_{\kappa, t}+\left[a_{t}+\bar{a}_{t}-\left(\Gamma_{t}+\bar{\Gamma}_{t}\right)\left(B_{t}+\bar{B}_{t}\right)^{-1}\left(b_{t}+\bar{b}_{t}\right)^{2}\right] \Lambda_{\kappa, t}=0 \\
\Lambda_{\kappa, T}=\kappa
\end{array}\right.
$$

which admit a unique solution, respectively. Plugging (30) into (23), one gets

$$
\begin{aligned}
u_{\kappa, t}= & -B_{t}^{-1}\left\{b_{t} \Gamma_{t} \hat{x}_{t}^{u_{\kappa}}+\left[b_{t} \bar{\Gamma}_{t}+\left(B_{t}+\bar{B}_{t}\right)^{-1}\left(B_{t} \bar{b}_{t}-\bar{B}_{t} b_{t}\right)\left(\Gamma_{t}+\bar{\Gamma}_{t}\right)\right] \mathbb{E} \hat{x}_{t}^{u_{\kappa}}\right. \\
& \left.+B_{t}\left(B_{t}+\bar{B}_{t}\right)^{-1}\left(b_{t}+\bar{b}_{t}\right) \Lambda_{\kappa, t}\right\},
\end{aligned}
$$

where $\Gamma, \bar{\Gamma}, \Lambda_{\kappa}$ and $\hat{x}^{u_{\kappa}}$ solve (31), (32), (33) and

$$
\left\{\begin{aligned}
d \hat{x}_{t}^{u_{\kappa}}= & \left\{\left(a_{t}-b_{t}^{2} B_{t}^{-1} \Gamma_{t}\right) \hat{x}_{t}^{u_{\kappa}}+\left[\bar{a}_{t}-\left(B_{t}+\bar{B}_{t}\right)^{-1}\left(b_{t}+\bar{b}_{t}\right)^{2} \bar{\Gamma}_{t}\right.\right. \\
& \left.-\left(B_{t}+\bar{B}_{t}\right)^{-1}\left(\bar{b}_{t}^{2}+2 b_{t} \bar{b}_{t}-B_{t}^{-1} \bar{B}_{t} b_{t}^{2}\right) \Gamma_{t}\right] \mathbb{E} \hat{x}_{t}^{u_{\kappa}} \\
& \left.-\left(B_{t}+\bar{B}_{t}\right)^{-1}\left(b_{t}+\bar{b}_{t}\right)^{2} \Lambda_{\kappa, t}\right\} d t \\
& +\left(\tilde{c}_{t}+\Sigma_{t} f_{t} h_{t}^{-1}\right) d \hat{\omega}_{t} \\
\hat{x}_{0}^{u_{\kappa}}= & \mu_{0}
\end{aligned}\right.
$$

respectively.

Step 3 Optimal control of Example 4.1.

Solving (27) and (28), one gets

$$
\beta_{\kappa, t}=\kappa e^{\int_{t}^{T} \rho_{s} d s},
$$




$$
\mathbb{E} \hat{x}_{t}^{u_{\kappa}}=\mu_{0} e^{\int_{0}^{t} \rho_{s} d s}-\kappa \int_{0}^{t}\left(B_{s}+\bar{B}_{s}\right)^{-1}\left(b_{s}+\bar{b}_{s}\right)^{2} e^{\int_{s}^{T} \rho_{r} d r+\int_{s}^{t} \rho_{r} d r} d s
$$

with

$$
\rho_{t}=a_{t}+\bar{a}_{t}-\left(B_{t}+\bar{B}_{t}\right)^{-1}\left(b_{t}+\bar{b}_{t}\right)^{2} \alpha_{t} .
$$

Recalling the terminal constraint (18), it yields

$$
\kappa_{0}=\frac{\mu_{0} e^{\int_{0}^{T} \rho_{t} d t}-\gamma}{\int_{0}^{T}\left(B_{t}+\bar{B}_{t}\right)^{-1}\left(b_{t}+\bar{b}_{t}\right)^{2} e^{2 \int_{t}^{T} \rho_{s} d s} d t} .
$$

Then Proposition 2.2 implies the desired conclusion. The above deduction is summarized as follows.

Proposition 4.1 The optimal feedback control of Example 4.1 is given by (34) with $\kappa$ being replaced by (35).

Example 4.2 In particular, if one lets the coefficients of (17), (19) and (20) $\bar{A}=\bar{B}=\bar{D}=\bar{a}=$ $\bar{b}=c=f=\bar{f}=g=\bar{g}=0$ on $[0, T]$, then Example 4.1 is reduced to an LQ optimal control with terminal constraint and complete information. Further, let $v \in L^{2}(0, T ; \mathbb{R})$. Since $v$ is deterministic, Proposition 4.1 implies that the optimal feedback control is

$$
u_{\kappa_{0}, t}=-B_{t}^{-1} b_{t}\left(\alpha_{t} \mathbb{E} x_{t}^{u_{\kappa_{0}}}+\beta_{\kappa_{0}, t}\right)
$$

where $\alpha, \beta_{\kappa_{0}}$ and $x^{u_{\kappa_{0}}}$ satisfy

$$
\begin{aligned}
& \left\{\begin{array}{l}
\dot{\alpha}_{t}+2 a_{t} \alpha_{t}-B_{t}^{-1} b_{t}^{2} \alpha_{t}^{2}+A_{t}=0, \\
\alpha_{T}=D
\end{array}\right. \\
& \beta_{\kappa_{0}, t}=\kappa_{0} e^{\int_{t}^{T}\left(a_{s}-B_{s}^{-1} b_{s}^{2} \alpha_{s}\right) d s},
\end{aligned}
$$

and

$$
\left\{\begin{array}{l}
d x_{t}^{u_{\kappa_{0}}}=\left(a_{t} x_{t}^{u_{\kappa_{0}}}-B_{t}^{-1} b_{t}^{2} \alpha_{t} \mathbb{E} x_{t}^{u_{\kappa_{0}}}-B_{t}^{-1} b_{t}^{2} \beta_{\kappa_{0}, t}\right) d t+\tilde{c}_{t} d \tilde{\omega}_{t}, \\
x_{0}^{u_{\kappa_{0}}}=\xi
\end{array}\right.
$$

with

$$
\kappa_{0}=\frac{\mu_{0} e^{\int_{0}^{T}\left(a_{t}-B_{t}^{-1} b_{t}^{2} \alpha_{t}\right) d t}}{\int_{0}^{T} B_{t}^{-1} b_{t}^{2} e^{2 \int_{t}^{T}\left(a_{s}-B_{s}^{-1} b_{s}^{2} \alpha_{s}\right) d s} d t},
$$

respectively.

Note that (36) is an SDE of mean-field type. It shows that one begins with a classical control system without mean-field term, but one ends up with a control system of meanfield type. This is a very interesting phenomenon indeed. 
Example 4.3 One denotes by $v$ the rate of capital withdrawal or injection of a firm, and by $x^{v}$ the cash-balance process on $[0, T]$. Assume that the liability of the firm is governed by

$$
-d \bar{L}_{t}^{v}=b_{t} v_{t} d t+c_{t} d \omega_{t}+\tilde{c}_{t} d \tilde{\omega}_{t},
$$

where $c_{t} d \omega_{t}$ and $\tilde{c}_{t} d \tilde{\omega}_{t}$ describe the liability risk. Assume that the firm owns an initial investment $\xi$, and only invests in a money account with compounded interest rate $a$. Then the cash-balance is denoted by

$$
x_{t}^{v}=e^{\int_{0}^{t} a_{s} d s}\left(\xi-\int_{0}^{t} e^{-\int_{0}^{s} a_{r} d r} d \bar{L}_{s}^{v}\right),
$$

whose differential form is the same as (6) with $\bar{a}=\bar{b}=0$. Due to the discreteness of the account information, the firm partially observes the cash-balance by the corresponding stock price

$$
\left\{\begin{array}{l}
d S_{t}^{v}=S_{t}^{v}\left[\left(f_{t} x_{t}^{v}+g_{t}+\frac{1}{2} h_{t}^{2}\right) d t+h_{t} d \tilde{\omega}_{t}\right] \\
S_{0}^{v}=1
\end{array}\right.
$$

Set

$$
y_{t}^{\nu}=\log S_{t}^{\nu} .
$$

It follows from Itô's formula that $y^{v}$ is governed by (7) with $\bar{f}_{t}=\bar{g}_{t}=0$. The firm hopes to find a suitable $v$ such that

$$
\mathcal{J}[v]=\frac{1}{2} \mathbb{E}\left[\int_{0}^{T} v_{t}^{2} d t+\left(x_{T}^{\nu}-\mathbb{E} x_{T}^{\nu}\right)^{2}\right]
$$

is minimized over $\mathcal{U}_{\text {ad }}^{c}$ with terminal constraint (18). The model implies that the firm wants to minimize the risk of $x_{T}^{v}$ and $v$ under a fixed terminal cash-balance level. Since

$$
\mathbb{E}\left(x_{T}^{v}-\mathbb{E} x_{T}^{v}\right)^{2}=\mathbb{E}\left(x_{T}^{v}\right)^{2}-\left(\mathbb{E} x_{T}^{v}\right)^{2},
$$

Example 4.3 is also a special case of Example 4.1. The following result is an immediate one of Proposition 4.1.

Corollary 4.1 The optimal rate of capital withdrawal or injection of the firm is

$$
u_{\kappa_{0}, t}=-b_{t}\left(\Gamma_{t} \hat{x}_{t}^{u_{\kappa_{0}}}+\bar{\Gamma}_{t} \mathbb{E} \hat{x}_{t}^{u_{\kappa_{0}}}+\Lambda_{\kappa_{0}, t}\right),
$$

where $\Gamma, \bar{\Gamma}, \Lambda$ and $\hat{x}^{u_{k_{0}}}$ are the solutions to

$$
\begin{aligned}
& \left\{\begin{array}{l}
\dot{\Gamma}_{t}+2 a_{t} \Gamma_{t}-b_{t}^{2} \Gamma_{t}^{2}=0, \\
\Gamma_{T}=1,
\end{array}\right. \\
& \left\{\begin{array}{l}
\dot{\bar{\Gamma}}_{t}+2\left(a_{t}-b_{t}^{2} \Gamma_{t}\right) \bar{\Gamma}_{t}-b_{t}^{2} \bar{\Gamma}_{t}^{2}=0, \\
\bar{\Gamma}_{T}=-1,
\end{array}\right.
\end{aligned}
$$




$$
\left\{\begin{array}{l}
\dot{\Lambda}_{\kappa_{0}, t}+\left[a_{t}-\left(\Gamma_{t}+\bar{\Gamma}_{t}\right) b_{t}^{2}\right] \Lambda_{\kappa_{0}, t}=0 \\
\Lambda_{\kappa_{0}, T}=\kappa_{0}
\end{array}\right.
$$

and

$$
\left\{\begin{array}{l}
d \hat{x}_{t}^{u_{\kappa_{0}}}=\left[\left(a_{t}-b_{t}^{2} \Gamma_{t}\right) \hat{x}_{t}^{u_{\kappa_{0}}}-b_{t}^{2} \bar{\Gamma}_{t} \mathbb{E} \hat{x}_{t}^{u_{\kappa_{0}}}-b_{t}^{2} \Lambda_{\kappa_{0}, t}\right] d t+\left(\tilde{c}_{t}+\Sigma_{t} f_{t} h_{t}^{-1}\right) d \hat{\omega}_{t}, \\
\hat{x}_{0}^{u_{\kappa_{0}}}=\mu_{0}
\end{array}\right.
$$

with

$$
\kappa_{0}=\frac{\mu_{0} e^{\int_{0}^{T} a_{t} d t}-\gamma}{\int_{0}^{T} b_{t}^{2} e^{2 \int_{t}^{T} a_{s} d s} d t}
$$

One remarks that the model in Example 4.3 is inspired by Huang et al. [18], where the variance of $v$ does not enter the performance functional.

\section{Concluding remarks}

This paper has studied an optimal control problem driven by SDE of mean-field tpye, where the drift coefficient of observation equation is linear with respect to the state, the control and their expectations, and the observation equation is explicitly dependent on the control. This framework covers many more financial models including [18], but it causes trouble in addressing the control problem. This trouble has been solved by the backward separation method with a decomposition technique. The results obtained here partially improve those of [3-6].

The traditional separation principle method is also applicable to study Problem (TC). However, the traditional method cannot offer a valid way to derive an optimality condition of Problem (TC). The reason is listed below. Let $\varrho(t, x)$ be the conditional density of $x_{t}^{v}$, given the observable filtration $\mathcal{F}_{t}^{y^{\nu}}$. Similar to Theorem 2.3 in [2], one gets

$$
\left\{\begin{aligned}
d \varrho(t, x)= & \left\{\frac{1}{2}\left(c_{t}^{2}+\tilde{c}_{t}^{2}\right) \frac{\partial^{2}}{\partial x^{2}} \varrho(t, x)+\left[a_{t} \varrho(t, x)+\left(a_{t} x+\bar{a}_{t} \mathbb{E} x_{t}^{v}+b_{t} v_{t}+\bar{b}_{t} \mathbb{E} x_{t}^{v}\right) \frac{\partial}{\partial x} \varrho(t, x)\right]\right\} d t \\
& +\left[f_{t} \varrho(t, x)\left(x-\int_{-\infty}^{+\infty} \chi \varrho(t, \chi) d \chi\right)-\tilde{c}_{t} \frac{\partial}{\partial x} \varrho(t, x)\right] h_{t} d \hat{\omega}_{t}, \\
\varrho(0, x)= & \frac{1}{\sqrt{2 \pi} \sigma_{0}} e^{-\frac{\left(x-\mu_{0}\right)^{2}}{2 \sigma_{0}^{2}}}, \quad(t, x) \in[0, T] \times \mathbb{R}
\end{aligned}\right.
$$

where

$$
\hat{\omega}_{t}=\int_{0}^{t} \frac{1}{h_{s}}\left[d y_{s}^{v}-\int_{-\infty}^{+\infty}\left(f_{s} \chi+\bar{f}_{s} \mathbb{E} x_{s}^{v}+g_{s} v_{s}+\bar{g}_{s} \mathbb{E} v_{s}\right) \varrho(s, \chi) d \chi d s\right]
$$

is an $\mathcal{F}_{t}^{y^{v}}$-adapted and $\mathbb{R}$-valued standard Brownian motion, and hence, the cost functional (9) is rewritten as

$$
\mathcal{J}[v]=\mathbb{E}\left[\int_{0}^{T} \int_{-\infty}^{+\infty} l\left(t, x, \mathbb{E} x_{t}^{v}, v_{t}, \mathbb{E} v_{t}\right) \varrho(t, x) d t+\phi\left(x, \mathbb{E} x_{T}^{v}\right) \varrho(T, x)\right] .
$$

This is an optimal control problem driven by stochastic partial differential equation with complete information. To obtain the optimality condition of the control problem, lots of stochastic calculuses on partial differential equation should be needed. Then it seems that the traditional method is not as effective as the case of Sects. 3 and 4 in this paper. 


\section{Appendix}

One presents three lemmas first, and then one gives a proof of Proposition 2.1.

Lemma A1 For any $v_{j} \in L_{\mathcal{F}}^{2}(0, T ; \mathbb{R}), j=1,2$, there is a constant $C>0$ such that

$$
\mathbb{E} \sup _{0 \leq t \leq T}\left|x_{t}^{v_{1}}-x_{t}^{v_{2}}\right|^{2} \leq C \mathbb{E} \int_{0}^{T}\left|v_{1, t}-v_{2, t}\right|^{2} d t
$$

Proof The estimate is obtained by Itô's formula and Hölder's inequality. The details of the proof are omitted to save space.

Lemma A2 For any $v, v_{j} \in \mathcal{U}_{\mathrm{ad}}, j=1,2, \ldots$,

$$
\lim _{j \rightarrow+\infty} J_{\kappa}\left[v_{j}\right]=J_{\kappa}[v]
$$

Proof Using Taylor's expansion, Hölder's inequality and Lemma A1, one gets

$$
\begin{aligned}
&\left|\mathbb{E} \int_{0}^{T} l\left(\Theta_{t}^{v_{j}}\right) d t-\mathbb{E} \int_{0}^{T} l\left(\Theta_{t}^{v}\right) d t\right| \\
&=\mid \mathbb{E} \int_{0}^{T}\left[\int_{0}^{1} l_{x}\left(\Upsilon_{t}^{\theta}\right) d \theta\left(x_{t}^{v_{j}}-x_{t}^{v}\right)+\int_{0}^{1} l_{\bar{x}}\left(\Upsilon_{t}^{\theta}\right) d \theta \mathbb{E}\left(x_{t}^{v_{j}}-x_{t}^{v}\right)\right. \\
&\left.+\int_{0}^{1} l_{v}\left(\Upsilon_{t}^{\theta}\right) d \theta\left(v_{j, t}-v_{t}\right)+\int_{0}^{1} l_{v}\left(\Upsilon_{t}^{\theta}\right) d \theta \mathbb{E}\left(v_{j, t}-v_{t}\right)\right] \mid \\
& \leq C \mathbb{E} \int_{0}^{T}\left(1+\left|x_{t}^{v_{j}}\right|+\left|x_{t}^{v}\right|+\mathbb{E}\left|x_{t}^{\nu_{j}}\right|+\mathbb{E}\left|x_{t}^{v}\right|+\left|v_{j, t}\right|+\left|v_{t}\right|+\mathbb{E}\left|v_{j, t}\right|+\mathbb{E}\left|v_{t}\right|\right) \\
& \times\left(\left|x_{t}^{\nu_{j}}-x_{t}^{v}\right|+\left|\mathbb{E} x_{t}^{v_{j}}-\mathbb{E} x_{t}^{v}\right|+\left|v_{j, t}-v_{t}\right|+\left|\mathbb{E} v_{j, t}-\mathbb{E} v_{t}\right|\right) d t \\
& \leq C \sqrt{\mathbb{E} \int_{0}^{T}\left(1+\left|x_{t}^{v_{j}}\right|^{2}+\left|x_{t}^{v}\right|^{2}+\mathbb{E}\left|x_{t}^{\nu_{j}}\right|^{2}+\mathbb{E}\left|x_{t}^{v}\right|^{2}+\left|v_{j, t}\right|^{2}+\left|v_{t}\right|^{2}+\mathbb{E}\left|v_{j, t}\right|^{2}+\mathbb{E}\left|v_{t}\right|^{2}\right) d t} \\
& \times\left(\sqrt{\mathbb{E} \sup _{0 \leq t \leq T}\left|x_{t}^{v_{j}}-x_{t}^{v}\right|^{2}}+\sqrt{\mathbb{E} \int_{0}^{T}\left|v_{j, t}-v_{t}\right|^{2} d t}\right) \rightarrow 0
\end{aligned}
$$

as $j \rightarrow+\infty$. Here $C>0$ is a constant which can be different from line to line. Similarly, one has

$$
\mathbb{E} \phi\left(\Xi_{T}^{v_{j}}\right) \rightarrow \mathbb{E} \phi\left(\Xi_{T}^{v}\right), \quad \mathbb{E} \varphi\left(\Xi_{T}^{v_{j}}\right) \rightarrow \mathbb{E} \varphi\left(\Xi_{T}^{v}\right)
$$

with $j \rightarrow+\infty$. Then the proof is complete.

Lemma $\mathbf{A} 3 \mathcal{U}_{\mathrm{ad}}$ is dense in $\mathcal{U}_{\mathrm{ad}}^{0}$.

Proof For any $v \in \mathcal{U}_{\mathrm{ad}}^{0}$, one defines a family of controls by

$$
v_{j, t}= \begin{cases}v, & \text { for } 0 \leq t \leq \delta_{j}, \\ \frac{1}{\delta j} \int_{(i-1) \delta_{j}}^{i \delta_{j}} v_{s} d s, & \text { for } i \delta_{j}<t \leq(i+1) \delta_{j}\end{cases}
$$


where $v \in U, i, j$ are natural numbers, $1 \leq i \leq j-1$, and $\delta_{j}=T / j$. Similar to [8], one proves that (i) $v_{j} \in \mathcal{U}_{\mathrm{ad}}$ for any $j$, and (ii) $v_{j} \rightarrow v$ as $j \rightarrow+\infty$ in $L_{\mathcal{F}^{0}}^{2}(0, T ; U)$. Thus the proof is complete.

Proof of Proposition 2.1 From the definition of decision set, it is easy to see

$$
\inf _{v^{\prime} \in \mathcal{U}_{\mathrm{ad}}} J_{\kappa}\left[v^{\prime}\right] \geq \inf _{v \in \mathcal{U}_{\mathrm{ad}}^{0}} J_{\kappa}[v]
$$

Then one only needs to prove the reverse inequality. Since $v_{j}$ introduced in Lemma A3 is an element of $\mathcal{U}_{\mathrm{ad}}$,

$$
J_{\kappa}\left[v_{j}\right] \geq \inf _{v^{\prime} \in \mathcal{U}_{\mathrm{ad}}} J_{\kappa}\left[v^{\prime}\right]
$$

and, consequently, it follows from Lemma A2 that

$$
J_{\kappa}[v]=\lim _{j \rightarrow+\infty} J_{\kappa}\left[v_{j}\right] \geq \inf _{v^{\prime} \in \mathcal{U}_{\mathrm{ad}}} J_{\kappa}\left[v^{\prime}\right]
$$

Then the arbitrariness of $v$ implies the desired result.

Proof of Proposition 2.2 According to the definition of optimal control and value function, the desired conclusion is drawn by using the idea introduced in Chap. 11 of Øksendal [19]. One drops the details of the proof for saving space.

Funding

This work is supported in part by the NSF of China under Grant No. 61603219, and by the Shandong Jiaotong University Climbing Research Innovation Team Program.

\section{Abbreviations}

SDE, stochastic differential equation; LQ, linear-quadratic; TC, terminal constraint.

Availability of data and materials

Not applicable.

Competing interests

The author declares that there is no conflict of interests regarding the publication of this paper.

Author's contributions

The author read and approved the final manuscript.

\section{Publisher's Note}

Springer Nature remains neutral with regard to jurisdictional claims in published maps and institutional affiliations.

Received: 26 September 2018 Accepted: 18 February 2019 Published online: 27 April 2019

\section{References}

1. Wang, G.C., Wu, Z:: Kalman-Bucy filtering equations of forward and backward stochastic systems and applications to recursive optimal control problems. J. Math. Anal. Appl. 342, 1280-1296 (2008)

2. Wang, G.C., Wu, Z., Xiong, J.: An Introduction to Optimal Control of FBSDE with Incomplete Information. Springer, Cham (2018)

3. Wang, G.C., Zhang, C.H., Zhang, W.H.: Stochastic maximum principle for mean-field type optimal control with partial information. IEEE Trans. Autom. Control 59, 522-528 (2014)

4. Zhang, H.Y.: A necessary condition for mean-field type stochastic differential equations with correlated state and observation noises. J. Ind. Manag. Optim. 12, 1287-1301 (2016)

5. Ma, H.P., Liu, B.: Optimal control problem for risk-sensitive mean-field stochastic delay differential equation with partial information. Asian J. Control 19, 2097-2115 (2017)

6. Buckdahn, R., Li, J., Ma, J.: A mean-field stochastic control problem with partial observations. Ann. Appl. Probab. 27, $3201-3245$ (2017) 
7. Wang, G.C., Wu, Z., Xiong, J.: Maximum principles for forward-backward stochastic control systems with correlated state and observation noises. SIAM J. Control Optim. 51, 491-524 (2013)

8. Wang, G.C., Wu, Z., Xiong, J.: A linear-quadratic optimal control problem of forward-backward stochastic differentia equations with partial information. IEEE Trans. Autom. Control 60, 2904-2916 (2015)

9. Wang, G.C., Xiao, H., Xing, G.J.: An optimal control problem for mean-field forward-backward stochastic differential equation with noisy observation. Automatica 86, 104-109 (2017)

10. Meyer-Brandis, T., Øksendal, B., Zhou, X.Y.: A mean-field stochastic maximum principle via Malliavin calculus. Stochastics 84, 643-666 (2012)

11. Elliott, R., Li, X., Ni, Y.H.: Discrete time mean-field stochastic linear quadratic optimal control problems. Automatica 49, 3222-3233 (2013)

12. Yong, J.M.: Linear-quadratic optimal control problems for mean-field stochastic differential equations. SIAM J. Control Optim. 51, 2809-2838 (2013)

13. Hafayed, H., Abbas, S.: On near-optimal mean-field stochastic singular controls: necessary and sufficient conditions for near-optimality. J. Optim. Theory Appl. 160, 778-808 (2014)

14. Ni, Y.H., Zhang, J.F., Li, X.: Indefinite mean-field stochastic linear-quadratic optimal control. IEEE Trans. Autom. Control 60, 1786-1800 (2015)

15. Hafayed, M., Abba, A., Abbas, S.: On partial-information optimal singular control problem for mean-field stochastic differential equations driven by teugels martingales measures. Int. J. Control 89, 397-410 (2016)

16. Xiong, J.: An Introduction to Stochastic Filtering Theory. Oxford University Press, London (2008)

17. Peng, S.G., Wu, Z.: Fully coupled forward-backward stochastic differential equations and applications to optimal control. SIAM J. Control Optim. 37, 825-843 (1999)

18. Huang, J.H., Wang, G.C., Wu, Z.: Optimal premium policy of an insurance firm: full and partial information. Insur. Math. Econ. 47, 208-215 (2010)

19. Øksendal, B.: Stochastic Differential Equations: An Introduction with Applications. Springer, New York (1998)

\section{Submit your manuscript to a SpringerOpen ${ }^{\circ}$ journal and benefit from:}

- Convenient online submission

- Rigorous peer review

- Open access: articles freely available online

- High visibility within the field

- Retaining the copyright to your article

Submit your next manuscript at $\boldsymbol{\nabla}$ springeropen.com 